\title{
Development of a Genetic Algorithm for Evaluating the Performance of Overhead Power Distribution Lines and Proposing Solutions to Improve Distribution Line Safety
}

\author{
Fabio Romero ${ }^{1}$, Alden U. Antunes ${ }^{1}$, Dario Takahata ${ }^{1}$, André Meffe ${ }^{1}$, Carlos C. B. Oliveira ${ }^{1}$, Fernando L. Lange ${ }^{1}$ \\ and Hamilton Souza ${ }^{2}$ \\ 1. Department of Engineering Research and Development, Daimon Engineering \& Systems, São Paulo 01310200, Brazil \\ 2. Department of Engineering, AES Eletropaulo Distribution Utility, São Paulo 06460040, Brazil
}

Received: July 08, 2014 / Accepted: September 04, 2014 / Published: January 31, 2015.

\begin{abstract}
This paper aims to present and discuss the use of a power flow methodology based on Gauss elimination method to evaluate the performance of distribution network taking into account the neutral conductor absence at specific sections, and a development of a methodology based on GA (genetic algorithm) capable of evaluating alternative solutions in different bars of the feeder, in order to propose appropriate solutions to improve the distribution network safety. Besides the technical aspects, the proposed GA methodology takes into account the economic feasibility analysis. The results of power flow simulations have shown that the presence of single-phase transformers along with the absence of the neutral conductor at specific sections of the MV (medium voltage) network may increase the Vng (neutral-to-ground voltage) levels of the feeders involved, jeopardizing the system's safety. On the other hand, the solutions proposed by the GA methodology may reduce the network Vng levels and improve the safety conditions, providing values close to the ones found before the neutral conductor theft.
\end{abstract}

Key words: Genetic algorithm, power flow, distribution line safety, power flow simulation.

\section{Introduction}

The performance evaluation of MV (medium voltage) systems has a great importance for utilities for planning and operation of distribution network purposes. The occurrence of neutral conductor theft has been increasing due to the easiness associated with the reselling of the copper and aluminium conductors at the black market. The unpredictability of this action may cause undesirable consequences to the electric utilities, such as impacts at the energy quality, increment of expenses and time for the maintenance crews to repair and/or reinstall the conductors, equipment damages, increase of the step and touch potentials etc..

Corresponding author: Fabio Romero, engineer, research fields: electrical power distribution planning, distribution losses, smart grid, protection of distribution lines, grounding, lightning electromagnetic fields, and power energy quality. E-mail: fabio.romero@daimon.com.br.
AES (Applied Energy Service) Eletropaulo (Brazil) has been suffering the aforementioned setbacks due to the absence of neutral conductor wires in its electric distribution network. The Utility is responsible for supplying about 6.3 million customers in the State of Sao Paulo, Brazil. The aim of this work is to present a genetic algorithm methodology to evaluate some alternatives along the MV network, in order to recommend appropriate solutions to reduce the Vng (neutral-to-ground voltage) levels and, consequently, improve the network safety. The power flow analysis, based on Gauss elimination method [1,2], evaluates the impact of neutral conductor absence at specific sections of the network regarding the performance and safety of distribution network, whereas, the GA (genetic algorithm) methodology proposes technical-economic solutions to mitigate the damages 
due to the neutral conductors theft.

The analyses presented in this paper have been part of the activities of an R \& D (Research and Development) Project carried out in partnership with AES Eletropaulo and the results have been applied in two different feeders of the utility.

\section{System Summarized Description}

AES Eletropaulo's distribution system is characterized by the presence of a common neutral conductor for the MV (medium voltage) and LV (low-voltage) circuits, multi-grounded at every $300 \mathrm{~m}$ and at every pole where there is an equipment installed (distribution transformers, reclosers, capacitor banks, voltage regulators etc.). The common multi-grounded neutral is employed in order to provide a low impedance path back to the substation [3].

The Gauss Elimination Method [1,2] and GA methodology have been implemented in the Interplan software [4] and the simulations were performed for the feeder RGR-104, due to the high incidence of its neutral conductor theft. Feeder RGR-104 is a $13.2 \mathrm{kV}$ circuit with $51 \mathrm{~km}$ long and, according to AES Eletropaulo, the neutral conductor is absent in about $20 \%$ of the total length.

Neutral conductors installed are 3/0 AWG (American Wire Gauge) aluminium wire at the main feeder and 1/0 AWG aluminium wire at the laterals.

Feeder RGR-104 is characterized by possessing $Y-\Delta$ (wye-delta) transformers, multigrounded neutral at every $300 \mathrm{~m}$ and at every pole where there is an electric distribution equipment installed, and also at poles adjacent to equipment. To evaluate the feeder's performance, simulations through Interplan power flow software have been employed, using as input, feeder RGR-104's geo-referenced data and phase and neutral current measurements at the substation. The methodology adopted in order to calculate the power flow allows, as well as other parameters, the phase and neutral current, neutral-ground voltage and electrical losses to be simulated. Table 1 shows the measured
Table 1 Measured and simulated values of phase and neutral currents at the substation of feeder RGR-104.

\begin{tabular}{llll}
\hline$I_{D}(\mathrm{~A})$ & $I_{E}(\mathrm{~A})$ & $I_{F}(\mathrm{~A})$ & $I_{N}(\mathrm{~A})$ \\
\hline 445 & 452 & 417 & 32 \\
\hline
\end{tabular}

values of phase and neutral currents at the substation of the feeder RGR-104.

The contributions for the ground resistances of the consumers' pole grounding and adjacent feeders are not considered in the simulations. Therefore, unless otherwise indicated and based on measurements along the feeder, the average ground resistivity $(\rho g)$ has been assumed to be $300 \Omega . \mathrm{m}$ and the average $R g$ (ground resistance) to be $50 \Omega$ for grounded poles.

The analysis with the GA proposed methodology takes into account the alternative solutions presented in the Table 2.

\section{GA (Genetic Algorithm) Methodology}

A methodology that uses the technique of GA [1] has been proposed, based on research of optimization methods for technical and economical assessments, and on research of alternative solutions to mitigate the problems due to the theft of neutral conductors in distribution networks.

GA methodology is a particular class of evolutionary algorithms that uses techniques inspired by evolutionary biology of Darwin's natural selection. The elements, which define a state of the problem from a population, are represented by chromosomes. These, in turn, are represented by genes. Thus, using some concepts of inheritance as crossover, mutation and natural selection of the fittest ones after a few generations, one expects to find the best solutions.

GA methodology is based on a combinatorial analysis, in which one seeks an optimal solution. The basic principle of GA technique is the adaptation of the population due to the inheritance of good genes of previous generations, and the good characteristics of individuals will be passed to future generations, according to the evaluation process. Fig. 1 shows the GA flowchart. 
Table 2 Alternative solutions evaluated by GA methodology to mitigate the damages due to the neutral conductor absence and improve the feeder safety condition.

\begin{tabular}{ll}
\hline Grounding configuration options & $\begin{array}{l}\text { Total installation cost } \\
\text { price (USD) }\end{array}$ \\
\hline 1,500 mm length four parallel rods & 130.00 \\
$1,500 \mathrm{~mm}$ length six parallel rods & 193.00 \\
2,400 mm length four parallel rods & 145.00 \\
2,400 mm length six parallel rods & 220.00 \\
\hline Neutral conductor options & $\begin{array}{l}\text { Total installation cost } \\
\text { price (USD/km) }\end{array}$ \\
\hline $\begin{array}{l}\text { Reinstallation of } 1 / 0 \mathrm{AWG} \\
\text { aluminium wire in the MV line }\end{array}$ & $1,450.00$ \\
sections without neutral conductors & \\
$\begin{array}{l}\text { Reinstallation of 3/0 AWG } \\
\text { aluminium wire in the MV line } \\
\text { sections without neutral conductors }\end{array}$ & $1,790.00$ \\
$\begin{array}{l}\text { Installation of bimetallic steel/ } \\
\text { aluminium wire 7N9 53\% IACS }\end{array}$ & $2,520.00$ \\
$\begin{array}{l}\text { Installation of bimetallic steel/ } \\
\text { aluminium wire 7N7 53\% IACS }\end{array}$ & $3,480.00$ \\
\hline IACS: International Annealed Cooper Standard.
\end{tabular}

IACS: International Annealed Cooper Standard.

One defines as an individual, the set of grounding solutions and conductors substitution of a specific configuration of the network, which will be represented by a finite size vector. The principle is analogous to the adaptation of living organisms in different environments. Initially, one draws the initial set of individuals, in which the population will be chosen, selecting the best suited beings, to apply later on the possibility of mutation and crossover. Finally, one assesses all individuals of this generation and, if a parameterized maximum number of generations are reached, one meets the most adapted individual. Otherwise, the cycle restarts from a selection of the best beings.

\subsection{Steps of GA Methodology for Determining} Adequate Solutions to Improve Network Safety

\subsubsection{Selection of Individuals}

The selection of individuals is carried out by employing the tournament method, i.e., one picks up randomly three individuals in the population and one selects only the most adapted individual.

\subsubsection{Mutation}

The aim of mutation is to generate new individuals with diverse characteristics, i.e., create greater variety of genes.

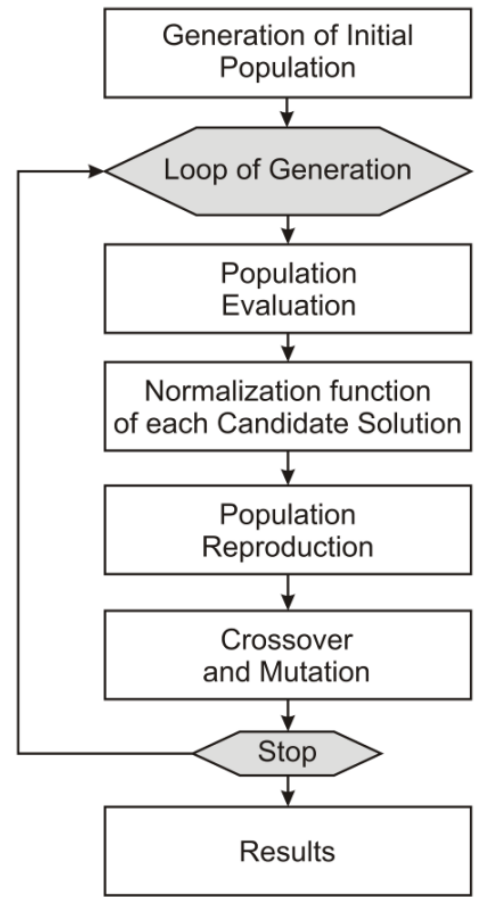

Fig. 1 Genetic algorithm flowchart.

\subsubsection{Recombination}

A recombination or crossover is required to increase the variety of individuals, or to increase the population of the most suited ones.

\subsubsection{Assessment}

The evaluation is performed through a merit index, which is composed by the grades assigned to the variables of "technical loss at the grounding" and cost of the proposed alternatives (Table 2). The technical loss variable is related to the value of $R_{g}$ and the leakage current through the grounding rod, i.e., the smaller the value of $R_{g}$ for a given leakage current, the smaller the technical loss in the ground; and consequently, the lesser the $V_{n g}$.

The merit index is assessed by the difference between the technical loss of the solution candidate individual and the initial technical loss of the individual (neutral stolen feeder configuration), represented by a certain cost to obtain this candidate, from the initial individual.

\subsection{Codification of the Individual}

The encoding of GA individuals is accomplished 
through a vector divided in two segments: grounding genes and conductor substitution genes. Fig. 2 presents a vector (chromosome) in its simplified version for a case of $n$ grounding configurations and $m$ neutral conductor substitution options.

One points out from Fig. 2 that each candidate (individual) is formed by a set of poles (with a specific $R_{g}$ ) and branches (with a specific neutral conductor).

The first part of the encoding vector is composed by the grounding configuration options (Table 2), in which each gene (grounded bar) will be set to the values " 0 " to " $x$ ", which are the grounding arrangement alternatives. Thus, contrary to conventional binary encoding, where there are only two possibilities in which the gene may worth, i.e., either " 0 " or " 1 ", each gene may alternatively be worth " 0 " to " $x$ ". In this paper, the genes (grounding bars, whether where there is equipment or where the bars are grounded every 300 $\mathrm{m})$ may have five grounding configuration options (Table 2):

- poles without grounding;

- 1,500 mm long four parallel rods;

- 1,500 mm long six parallel rods;

- 2,400 mm long four parallel rods;

- 2,400 mm long six parallel rods.

The second part of the encoding vector is composed by the neutral conductor options (Table 2), in which the options for each gene of conductor substitution (absent neutral conductor branch) will be set to the values "0" to " $y$ ", which are the conductor substitution alternatives. Thus, contrary to conventional binary encoding, where there are only two possibilities in which the gene may worth, i.e., either " 0 " or " 1 ", each gene may alternatively be worth " 0 " to " $y$ ". In this paper, the genes (absent neutral conductor branches) may only have five neutral conductor options:

- MV branch without neutral conductor;

- MV branch with 1/0 AWG aluminium wire;

- MV branch with 3/0 AWG aluminium wire;

- MV branch with bimetallic steel/aluminum wire 7N9 53\% IACS;

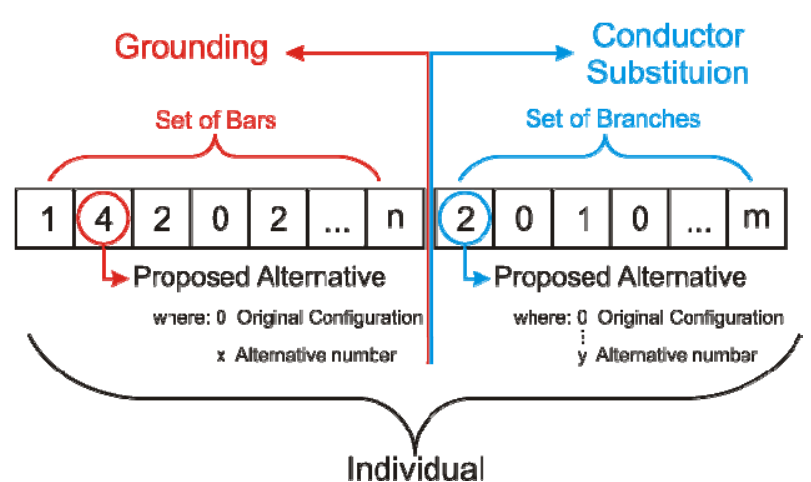

Fig. 2 Codification of a finite size vector (representing a chromosome).

- MV branch with bimetallic steel/aluminum wire 7N7 53\% IACS.

\subsection{Evaluation Function}

The GA should find the best solution to maximize the evaluation function feval (losses, cost) as follows:

$f_{\text {eval }}($ losses, $\cos t)=$

$\left(\frac{\text { Initial_Configuration_Losses }- \text { Analysed_Individual_Losses }}{\text { Instalation_Costs }}\right)$

where,

- Analysed Individual Losses: economic value of the grounding losses of the analyzed alternative, based on the study period (years), on the initial year energy cost (US\$/MWh), on the market growth rate (percentage per annum) and internal rate of return (percentage per annum);

- Initial_Configuration_Losses: economic value of grounding losses of the system original configuration, i.e., network with some branches without neutral conductors, adopting the same period and economic indices;

- Installation Costs: economic value of the chosen alternative, considering the same parameters aforementioned.

Fig. 3 shows the results of a simulation, with the proposed alternatives in Table 2.

Fig. 3 shows that the best alternative is the one which presents a grounding energy loss value around US $\$ 3,770.00$, and an installation cost of approximately US\$50,000.00 in a six-year period. 


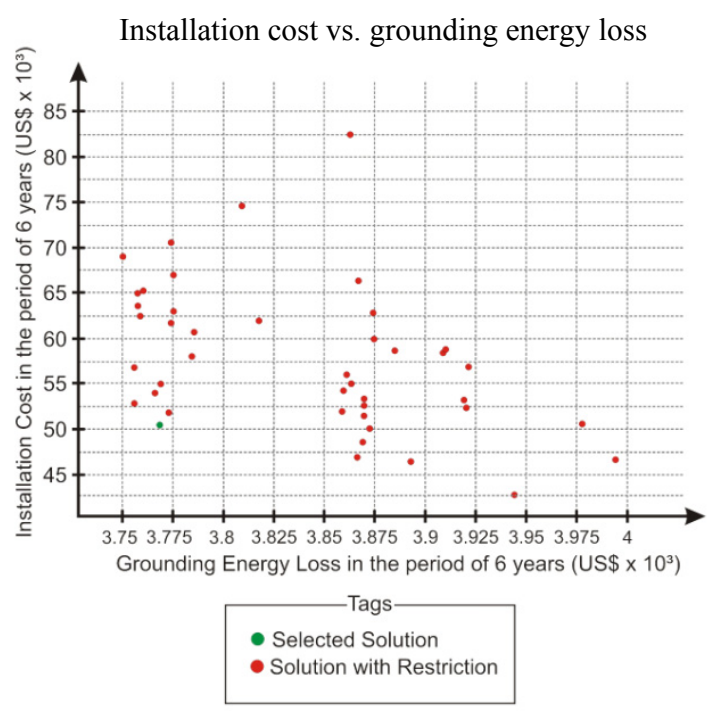

Fig. 3 Alternative solutions presented by GA proposed methodology.

\section{Conclusions}

This paper has evaluated the performance of MV distribution networks due to the lack of neutral conductors in some of its branches and proposed technical solutions to mitigate the damage caused by the operation of the network under these operative conditions. Feeder RGR-104 has been used to simulate due to its high incidence of neutral conductor theft.

To evaluate the performance of the feeder taking into account the network neutral conductor thefts, a new methodology for power flow method based on Gauss Elimination has been developed and implemented into Interplan software [4]. The simulation results show that the absence of neutral conductors, in conjunction with the imbalance caused by the wye transformer connection along the feeder, greatly increases the levels of neutral-ground voltage, decreasing the security of the network.

A new methodology based on GA has been developed to improve the network security, proposing appropriate solutions (including changes in grounding systems and replacement and/or installation of new conductors) to keep the network Vng levels within adequate technical and safety conditions.

As an alternative to eliminate the frequent thefts of aluminum neutral conductors in certain regions of the feeder, bimetallic conductors have been used in branches with high theft incidence.

The results presented in this paper may be used as reference for planning improvements in the performance of distribution networks. However, it should be stressed that the results tend to be conservative because one has neglected the influence of consumer's pole grounding and the groundings of adjacent feeders near the studied feeder, and also the variations in ground resistance and soil resistivity should be taken into account.

\section{References}

[1] Antunes, A. U., Méffe, A., Takahata, D., Kamikoga, D. H., Romero, F., and Lange. F. L. 2010. Specification of Power Flow Methodology and Technical Economic Analysis of Alternative Solutions to Mitigate Damages Due to Neutral Conductor's Theft. Technical report for AES Eletropaulo Distribution Utility, Sao Paulo, Brazil.

[2] Antunes, A. U., Antonelli, D., Nanni, M., and Romero, F. 2010. Specification Models for Power Flow Calculations. Technical report for AES Eletropaulo Distribution Utility, Sao Paulo, Brazil.

[3] Zipse, D. W. 2004. "The Hazardous Multigrounded Neutral Distribution System and Dangerous Stray Currents." In Proceedings of Petroleum and Chemical Industry Conference IEEE, 23-45.

[4] Oliveira, C. C. B., Kagan, N., Guaraldo, J. C., El Hage, F. S., Meffe, A., and Filho, M. M. 2004. "Interplan-A Tool for Planning High, Medium and Low Voltage Networks." Presented at IEEE the Transmission and Distribution Conference and Exposition, Denver, USA. 